\title{
Flavour changing top decays in supersymmetric extensions of the standard model
}

\author{
G. M. de Divitiis, R. Petronzio and L. Silvestrini \\ Dipartimento di Fisica, Università di Roma Tor Vergata \\ and \\ INFN, Sezione di Roma II \\ Via della Ricerca Scientifica 1, 00133 Roma, Italy
}

April 4, 1997

\begin{abstract}
Flavour changing top decays $t \rightarrow c Z^{0}, t \rightarrow c g$ and $t \rightarrow c \gamma$ are predicted with invisible rates within the standard model and may represent a window on new physics. We consider these processes in supersymmetric extensions of the standard model and we show that observable rates can be obtained only if the SUSY breaking is non universal and flavour dependent.
\end{abstract}




\section{Introduction}

Flavour changing neutral currents (FCNC) in the Standard Model (SM) are absent at tree level and suppressed by the GIM mechanism at one loop. They are particularly sensitive to large mass splittings between quarks of different generations.

Within the SM, the absence of tree-level FCNC implies that inside the loop only charged currents can mediate the flavour change and therefore the large splitting of the third generation in the up sector can only be effective in processes with external $d$-type quarks.

The smallness of the mixing angles between the first two generations and the third one implies that the effects of a heavy top are particularly evident only in FCNC processes involving $b$ quarks, such as $B-\bar{B}$ mixing, radiative $B$ decays, etc.

The extensions of the standard model to multi-Higgs doublet schemes allow for the presence of tree level FCNC which can be tested also with external up-type quarks and in particular in the FCNC top decays. This study was pursued in refs. [1], where it was shown that one could achieve values of the branching ratios of the order of $10^{-6}$, i.e. orders of magnitude bigger than the standard model estimates of the order of $10^{-11}$.

Tree-level FCNC vertices are also present in supersymmetric extensions of the SM. In particular, in the Minimal Supersymmetric Standard Model (MSSM), i.e. in the supersymmetric extension of the SM with minimal particle content, FCNC vertices involving fermions, sfermions and gauginos arise because of the misalignment of fermion and sfermion low-energy mass matrices. By imposing R parity, these vertices can only contribute to FCNC quark decays at one loop level via penguin diagrams in which the intermediate particles are gluinost. The difference with SM W-mediated penguins, and with SUSY chargino-mediated penguins, is that gluino-mediated penguins involving external up-type quarks are proportional to the mass splitting between up-type squarks which closely follows the one of the corresponding non supersymmetric partners.

The purpose of this paper is to analyse neutral current top decays, $t \rightarrow c Z^{0}, t \rightarrow c g$ and $t \rightarrow c \gamma$, in supersymmetric extensions of the standard model.

We give the estimates for the MSSM with flavour-universal soft SUSY breaking terms at the GUT scale, and also for a more general class of models where such a flavour universality does not hold. The universal case was already discussed in the literature [2, 3]. In this case we have recomputed both charginos and gluinos contributions.

We disagree with the results of refs. [2, 3] mainly in the evaluation of the relevant SUSY mixing angles and in a detail of the actual calculation of the decay amplitude.

We find that the SUSY mixing angle between the second and the third generation has been over-estimated by at least one order of magnitude. We also

\footnotetext{
${ }^{1}$ We will neglect neutralino contributions, because they are in general suppressed by powers of $\alpha_{W} / \alpha_{s}$.
} 
find a difference in the result for the amplitude which can be traced back to the omission in previous papers of the diagrams involving a helicity flip in the gluino line, which dominate the branching ratios when the gluino mass gets large.

The signal for the case where the SUSY breaking is universal turns out too low to be detectable.

In the non-universal case, where the soft SUSY breaking is not flavour blind, the branching ratio can be as high as $10^{-5}$, which is maybe detectable. In this case the contributions from intermediate chargino exchange with respect to gluino exchange can be generally neglected. In Section 2 we review briefly those aspects of the MSSM which are relevant for FCNC. In section 3 we present the details of the calculation. The last section is devoted to the presentation of the results and to the conclusions.

\section{The MSSM}

The superpotential of the MSSM is given by:

$$
W=Y_{i j}^{u} Q_{i} H_{2} U_{j}^{c}+Y_{i j}^{d} Q_{i} H_{1} D_{j}^{c}+Y_{i j}^{\ell} L_{i} H_{1} E_{j}^{c}-\mu H_{1} H_{2},
$$

which is the R-parity conserving supersymmetric generalization of the Yukawa interactions of quarks and Higgs bosons in the SM.

The breaking of SUSY is accounted for by introducing at the GUT scale $M_{G}$ the following soft SUSY breaking terms:

$$
\begin{aligned}
-\mathcal{L}_{s}\left(M_{G}\right)= & \widetilde{Q}_{L}^{i^{\dagger}}\left(\bar{m}_{\widetilde{Q}_{L}}^{2}\right)_{i j} \widetilde{Q}_{L}^{j}+\widetilde{u}_{R}^{i^{\dagger}}\left(\bar{m}_{\widetilde{u}_{R}}^{2}\right)_{i j} \widetilde{u}_{R}^{j}+\widetilde{d}_{R}^{i^{\dagger}}\left(\bar{m}_{\widetilde{d}_{R}}^{2}\right)_{i j} \widetilde{d}_{R}^{j} \\
+ & \widetilde{L}_{L}^{i^{\dagger}}\left(\bar{m}_{\widetilde{L}_{L}}^{2}\right)_{i j} \widetilde{L}_{L}^{j}+\widetilde{\ell}_{R}^{\dagger}\left(\bar{m}_{\widetilde{\ell}_{R}}^{2}\right)_{i j} \widetilde{\ell}_{R}^{j}+\bar{m}_{1}^{2} h_{1}^{\dagger} h_{1}+\bar{m}_{2}^{2} h_{2}^{\dagger} h_{2} \\
+ & {\left[\bar{A}_{i j}^{u} \widetilde{Q}_{L}^{i} \tilde{u}_{R}^{j} h_{2}+\bar{A}_{i j}^{d} \widetilde{Q}_{L}^{i} \tilde{d}_{R}^{j} h_{1}+\bar{A}_{i j}^{\ell} \widetilde{L}_{L}^{i} \tilde{\ell}_{R}^{j} h_{1}+B \mu h_{1} h_{2}\right.} \\
& \left.+\frac{1}{2}\left(\bar{m}_{\tilde{g}} \tilde{g}^{T} C \tilde{g}+\bar{m}_{\widetilde{W}} \widetilde{W}^{T} C \widetilde{W}+\bar{m}_{\widetilde{B}} \widetilde{B}^{T} C \widetilde{B}\right)+\text { h. c. }\right]
\end{aligned}
$$

where $\mathrm{C}$ is the charge conjugation matrix and $i, j=1,2,3$.

We first consider a constrained version of the MSSM in which we impose universality of the soft breaking terms at the GUT scale:

$$
\begin{aligned}
& \left(\bar{m}_{\widetilde{Q}_{L}}^{2}\right)_{i j}=\left(\bar{m}_{\widetilde{u}_{R}}^{2}\right)_{i j}=\left(\bar{m}_{\widetilde{d}_{R}}^{2}\right)_{i j}=\left(\bar{m}_{\widetilde{L}_{L}}^{2}\right)_{i j}=\left(\bar{m}_{\widetilde{\ell}_{R}}^{2}\right)_{i j}=m_{0}^{2} \delta_{i j}, \\
& \bar{A}_{i j}^{u}=A_{0} Y_{i j}^{u} \quad \bar{A}_{i j}^{d}=A_{0} Y_{i j}^{d} \\
& \bar{m}_{\tilde{g}}=\bar{m}_{\widetilde{W}}^{\ell}=\bar{m}_{\widetilde{B}}=m_{1 / 2} .
\end{aligned}
$$

With these soft breaking terms, the model is defined by six parameters:

$$
m_{0}, \quad m_{1 / 2}, \quad A_{0}, \quad \tan \beta, \quad \mu \text { and } B .
$$

The request of radiative breaking of the electroweak symmetry reduces the parameters of the model to five:

$$
m_{0}, \quad m_{1 / 2}, \quad A_{0}, \quad \tan \beta \text { and } \operatorname{sign}(\mu) .
$$


The low-energy values of the couplings in eqs. (11) and (2) are obtained by solving the following renormalization group equations 4 - 6 :

$$
\begin{aligned}
\frac{\mathrm{d} \boldsymbol{Y}^{u}}{\mathrm{dt}} & =-\frac{1}{2}\left[3\left(\boldsymbol{Y}^{u} \boldsymbol{Y}^{u^{\dagger}}+\operatorname{Tr} \boldsymbol{Y}^{u} \boldsymbol{Y}^{u^{\dagger}}\right)+\boldsymbol{Y}^{d} \boldsymbol{Y}^{d^{\dagger}}-2 C_{i}^{\mathrm{u}} g_{i}^{2}\right] \boldsymbol{Y}^{u}, \\
\frac{\mathrm{d} \boldsymbol{A}^{u}}{\mathrm{dt}} & =-\frac{1}{2}\left[5 \boldsymbol{Y}^{u} \boldsymbol{Y}^{u^{\dagger}}+3 \operatorname{Tr} \boldsymbol{Y}^{u} \boldsymbol{Y}^{u^{\dagger}}+\boldsymbol{Y}^{d} \boldsymbol{Y}^{d^{\dagger}}-2 C_{i}^{\mathrm{u}} g_{i}^{2}\right] \boldsymbol{A}^{u} \\
& -\left[2 \boldsymbol{A}^{u} \boldsymbol{Y}^{u^{\dagger}}+3 \operatorname{Tr} \boldsymbol{A}^{u} \boldsymbol{Y}^{u^{\dagger}}+\boldsymbol{A}^{d} \boldsymbol{Y}^{d^{\dagger}}-2 C_{i}^{\mathrm{u}} g_{i}^{2} M_{i}\right] \boldsymbol{Y}^{u}, \\
\frac{\mathrm{d} \boldsymbol{m}_{\widetilde{Q}_{L}}^{2}}{\mathrm{dt}} & =-\frac{1}{2}\left[\left\{\boldsymbol{Y}^{u} \boldsymbol{Y}^{u^{\dagger}}+\boldsymbol{Y}^{d} \boldsymbol{Y}^{d^{\dagger}}, \boldsymbol{m}_{\widetilde{Q}_{L}}^{2}\right\}+2\left(m_{h_{u}}^{2} \boldsymbol{Y}^{u} \boldsymbol{Y}^{u^{\dagger}}+m_{h_{d}}^{2} \boldsymbol{Y}^{d} \boldsymbol{Y}^{d^{\dagger}}\right.\right. \\
& \left.\left.+\boldsymbol{Y}^{u} \boldsymbol{m}_{\tilde{u}_{R}}^{2} \boldsymbol{Y}^{u^{\dagger}}+\boldsymbol{Y}^{d} \boldsymbol{m}_{\tilde{d}_{R}}^{2} \boldsymbol{Y}^{d^{\dagger}}+\boldsymbol{A}^{u} \boldsymbol{A}^{u^{\dagger}}+\boldsymbol{A}^{d} \boldsymbol{A}^{d^{\dagger}}\right)\right]+4 C_{i}^{Q_{L}} g_{i}^{2} M_{i}^{2}, \\
\frac{\mathrm{d} \boldsymbol{m}_{\tilde{u}_{R}}^{2}}{\mathrm{dt}} & =-\left[\left\{\boldsymbol{Y}^{u} \boldsymbol{Y}^{u^{\dagger}}, \boldsymbol{m}_{\tilde{u}_{R}}^{2}\right\}+2\left(m_{h_{u}}^{2} \boldsymbol{Y}^{u} \boldsymbol{Y}^{u^{\dagger}}+\boldsymbol{Y}^{u} \boldsymbol{m}_{\tilde{u}_{R}}^{2} \boldsymbol{Y}^{u^{\dagger}}+\boldsymbol{A}^{u} \boldsymbol{A}^{u^{\dagger}}\right)\right] \\
& +4 C_{i}^{u} g_{i}^{2} M_{i}^{2}, \\
\frac{\mathrm{d} m_{h_{u}}^{2}}{\mathrm{dt}} & =-3 \operatorname{Tr}\left[\boldsymbol{Y}^{u}\left(\boldsymbol{m}_{\widetilde{Q}_{L}}^{2}+\boldsymbol{m}_{\tilde{u}_{R}}^{2}\right) \boldsymbol{Y}^{u^{\dagger}}+m_{h_{u}}^{2} \boldsymbol{Y}^{u} \boldsymbol{Y}^{u^{\dagger}}+\boldsymbol{A}^{u} \boldsymbol{A}^{u^{\dagger}}\right] \\
& +4 C_{i}^{h_{u}} g_{i}^{2} M_{i}^{2},
\end{aligned}
$$

where $t=1 /\left(16 \pi^{2}\right) \log \left(M_{G}^{2} / Q^{2}\right)$ is related to the running scale $Q^{2}$. One has also to consider the additional set obtained through the replacement $u \leftrightarrow d$. In eq. (6) we have neglected the leptonic contribution. We have denoted by $M_{i}$ the gaugino masses and by $C_{i}^{R}$ the quadratic casimir eigenvalues of $\mathrm{SU}(3), \mathrm{SU}(2)$ and $\mathrm{U}(1)$ for the representation $R$, with the further definition

$$
C_{i}^{\mathrm{u}}=C_{i}^{Q_{L}}+C_{i}^{u_{R}}+C_{i}^{h_{u}}, \quad C_{i}^{\mathrm{d}}=C_{i}^{Q_{L}}+C_{i}^{d_{R}}+C_{i}^{h_{d}} .
$$

At the electroweak scale, we choose a basis such that the Yukawa couplings of up-type quarks are flavour-diagonal,

$$
\boldsymbol{Y}^{u}=\boldsymbol{Y}_{D}^{u}, \quad \boldsymbol{Y}^{d}=\boldsymbol{K} \boldsymbol{Y}_{D}^{d}
$$

where

$$
\begin{aligned}
& \boldsymbol{m}_{D}^{u}=\operatorname{diag}\left(m_{u}, m_{c}, m_{t}\right)=\frac{v \sin \beta}{\sqrt{2}} \boldsymbol{Y}_{D}^{u} \\
& \boldsymbol{m}_{D}^{d}=\operatorname{diag}\left(m_{d}, m_{s}, m_{b}\right)=\frac{v \cos \beta}{\sqrt{2}} \boldsymbol{Y}_{D}^{d}
\end{aligned}
$$

$v=\sqrt{v_{1}^{2}+v_{2}^{2}}=246 \mathrm{GeV}$ and $\tan \beta=v_{2} / v_{1}$.

In the super-CKM basis, in which the quark-squark-gaugino couplings are equal to the quark-quark-gauge boson ones, the squark mass matrices at the electroweak scale are given by

$$
\boldsymbol{m}_{\tilde{u}}^{2}=\left(\begin{array}{cc}
\boldsymbol{m}_{\tilde{u}_{L L}}^{2} & \boldsymbol{m}_{\tilde{u}_{L R}}^{2} \\
\boldsymbol{m}_{\tilde{u}_{L R}}^{2} & \boldsymbol{m}_{\tilde{u}_{R R}}^{2}
\end{array}\right), \quad \boldsymbol{m}_{\tilde{d}}^{2}=\left(\begin{array}{cc}
\boldsymbol{m}_{\tilde{d}_{f L}}^{2} & \boldsymbol{m}_{\tilde{d}_{L R}}^{2} \\
\boldsymbol{m}_{\tilde{d}_{L R}}^{2} & \boldsymbol{m}_{\tilde{d}_{R R}}^{2}
\end{array}\right),
$$


where

$$
\begin{aligned}
\boldsymbol{m}_{\tilde{u}_{L L}}^{2} & =\boldsymbol{m}_{\widetilde{Q}_{L}}^{2}+\left(\boldsymbol{m}_{D}^{u}\right)^{2}+\frac{M_{Z}^{2}}{6}\left(3-4 \sin ^{2} \theta\right) \cos 2 \beta \\
\boldsymbol{m}_{\tilde{u}_{L R}}^{2} & =-\mu \boldsymbol{m}_{D}^{u} \cot \beta-\frac{v \sin \beta}{\sqrt{2}} \boldsymbol{A}^{u} \\
\boldsymbol{m}_{\tilde{u}_{R R}}^{2} & =\boldsymbol{m}_{\tilde{u}_{R}}^{2}+\left(\boldsymbol{m}_{D}^{u}\right)^{2}+\frac{2}{3} M_{Z}^{2} \sin ^{2} \theta \cos 2 \beta \\
\boldsymbol{m}_{\tilde{d}_{L L}}^{2} & =\boldsymbol{K}^{\dagger} \boldsymbol{m}_{\widetilde{Q}_{L}}^{2} \boldsymbol{K}+\left(\boldsymbol{m}_{D}^{d}\right)^{2}-\frac{M_{Z}^{2}}{6}\left(3-2 \sin ^{2} \theta\right) \cos 2 \beta \\
\boldsymbol{m}_{\tilde{d}_{L R}}^{2} & =-\mu \boldsymbol{m}_{D}^{d} \tan \beta-\frac{v \cos \beta}{\sqrt{2}} \boldsymbol{K}^{\dagger} \boldsymbol{A}^{d} \\
\boldsymbol{m}_{\tilde{d}_{R R}}^{2} & =\boldsymbol{m}_{\tilde{d}_{R}}^{2}+\left(\boldsymbol{m}_{D}^{u}\right)^{2}-\frac{1}{3} M_{Z}^{2} \sin ^{2} \theta \cos 2 \beta .
\end{aligned}
$$

In the low $\tan \beta$ regime, in which the effects of the Yukawa coupling of the bottom quark $Y_{b}$ can be neglected, the effect of $Y_{t}$ in the evolution of the soft breaking masses is to induce a mass splitting between squarks; however, the mass matrix for up-type squarks remains diagonal [5, 13]. On the other hand, for large $\tan \beta Y_{b}$ gets large and sizeable off-diagonal terms are induced by the evolution in $\boldsymbol{m}_{\tilde{u}}^{2}$. However, it is easy to see from eq. (6) that the off-diagonal terms between $\tilde{c}$ and $\tilde{t}$ are proportional to $K_{23}$, and therefore at most of the order of a few percents, to be compared with the value of $\pi / 6$ quoted in refs. [2, 3].

In figure 1 we report for different values of $\tan \beta$ the renormalization group evolution of the value of the ratio of the off-diagonal $\tilde{c}_{L}-\tilde{t}_{L}$ matrix element squared divided by the average diagonal matrix element squared, which is an estimate of the mixing angle in the mass eigenstate basis.

As we shall see in section 4 , such a small mixing angle renders the prediction of the constrained MSSM for rare top decays hard to detect. This is essentially because the only FCNC effects in the squark mass matrix are induced by the quark mass matrix, and are again protected by a GIM mechanism: they disappear when the down quark masses are degenerate.

By relaxing the universality constraints one introduces a substantial flavour mixing in the theory, resulting in large contributions to FCNC processes [8]-11]. There are strong constraints on non-universal soft breaking terms involving the first two families; however, the off-diagonal squark mass terms between $\tilde{c}$ and $\tilde{t}$ are unconstrained by available data on low-energy FCNC processes:2]. This means that we can envisage a situation in which there is a large mixing angle between $\tilde{c}$ and $\tilde{t}$, resulting in large constributions to rare top decays, as we shall see in section 4 , in which we consider generalizations of the MSSM with arbitrary mixing angles in this sector and nonuniversal gaugino masses.

\footnotetext{
${ }^{2}$ In principle, constraints on these mass terms could be obtained from a study of chargino contributions to $b \rightarrow s \gamma$ decay in non-universal SUSY [12].
} 


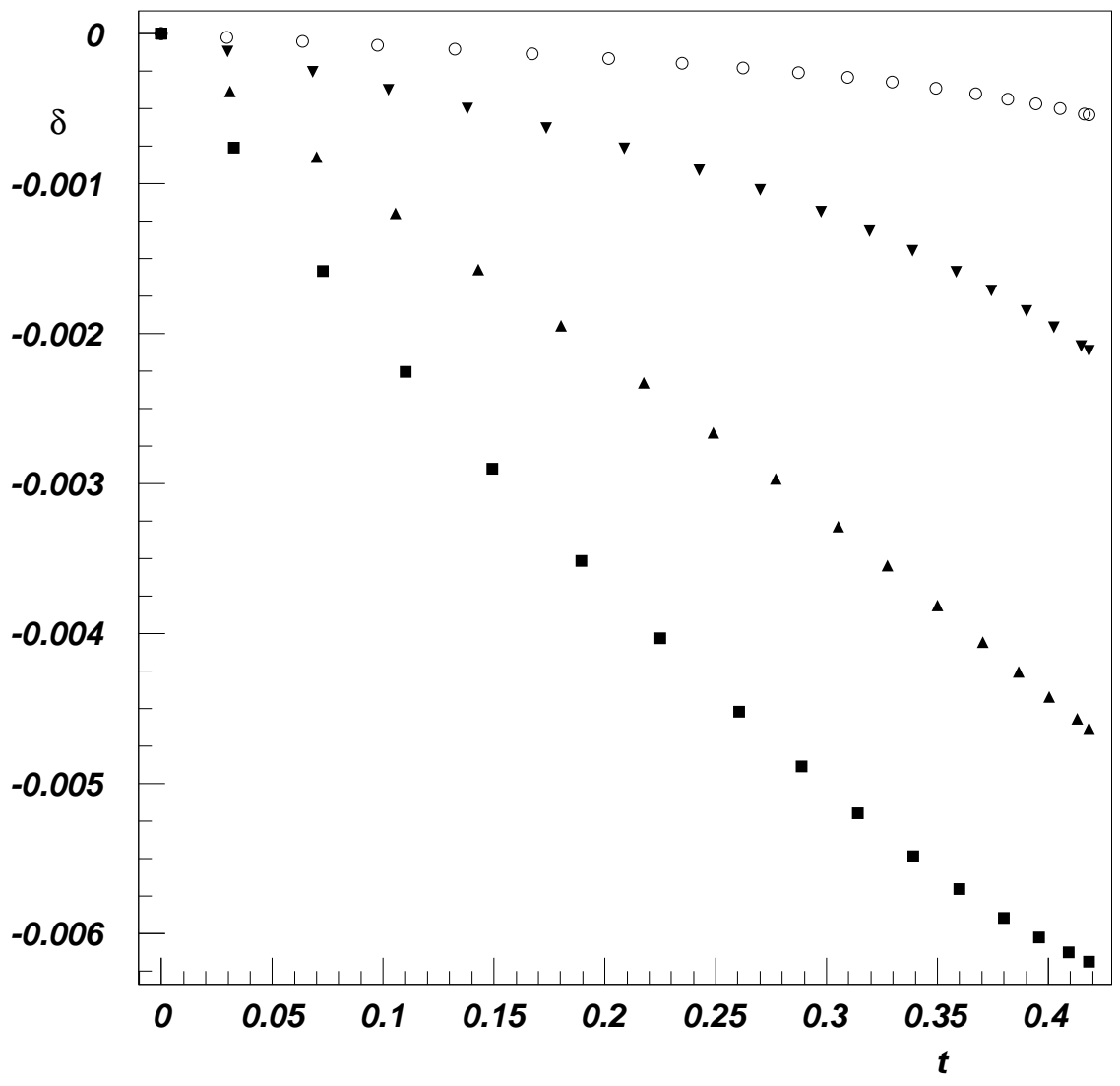

Figure 1: The renormalization group evolution of the ratio $\delta$ of the off diagonal element $\tilde{c}_{L}-\tilde{t}_{L}$ of the squared mass matrix over the average of the diagonal elements of the same matrix in the minimal SUSY model with universal coupling as a function ot $t=1 /\left(16 \pi^{2}\right) \log \left(M_{G}^{2} / Q^{2}\right)$. The four curves refer to different values of $\tan \beta: 10,20,30,35$ (from the upper to the lower). 

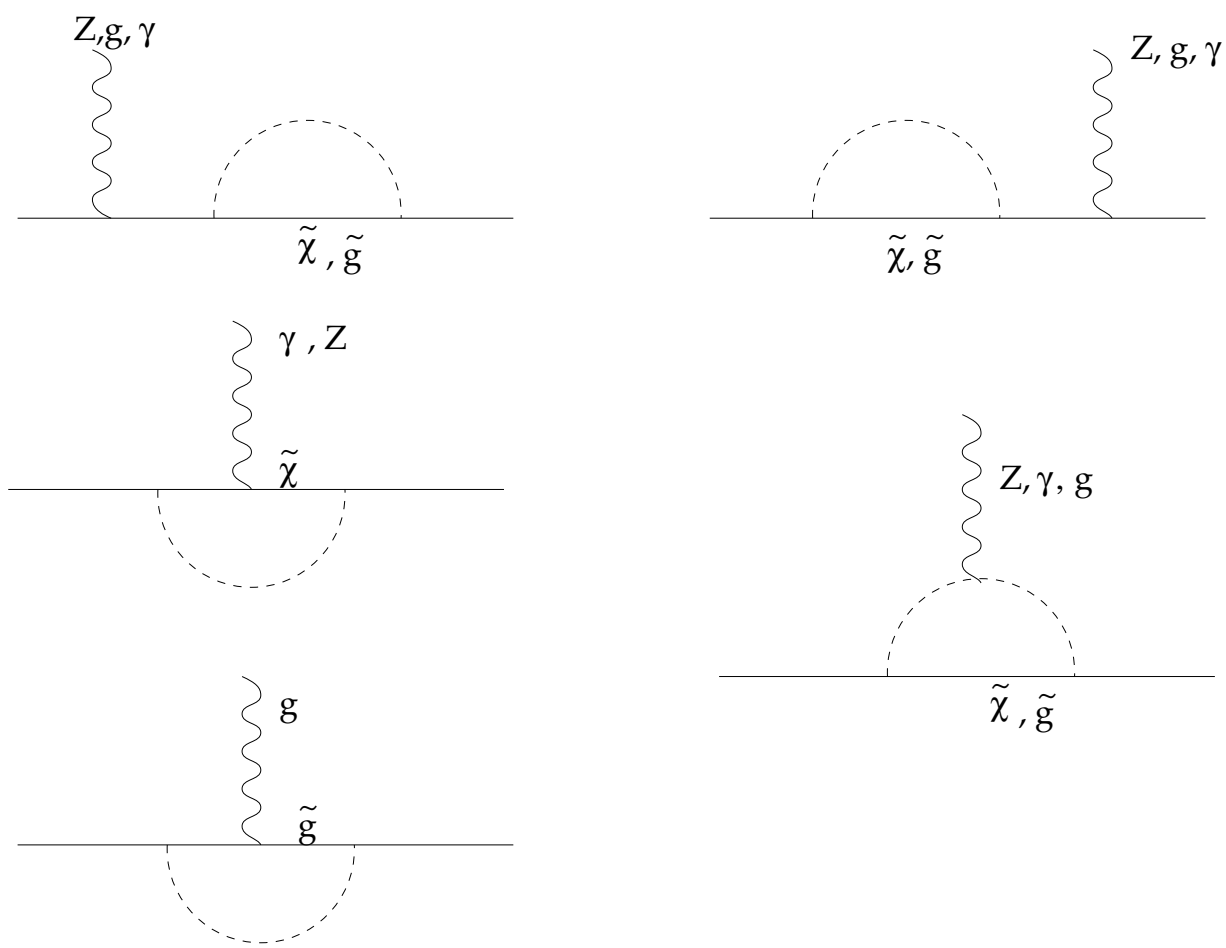

Figure 2: Diagrams for the processes $t \rightarrow c Z^{0}, t \rightarrow c g, t \rightarrow c \gamma$. Dashed lines are for squarks.

\section{The calculation}

The calculation was rederived independently.

The general diagrams contributing to top decays are given in figure 2 for the processes $t \rightarrow c Z^{0}, t \rightarrow c g, t \rightarrow c \gamma$.

When the flavour changing is a small effect, its contribution can be safely estimated in the "mass insertion" approximation [7]-[11], where the vertices are kept flavour conserving and the flavour off diagonal terms are treated perturbatively as mass insertions in the squark propagators. This approximation allows to make estimates which can be easily transferred from one model to another, but in general cannot be trusted when the perturbation is not small.

This applies in particular to the left-right top squark mixing: its main effect is to split considerably the corresponding mass eigenstates and to make the transition less affected by the GIM mechanism.

In general, there are three form factors, current conservation restricts them to only two for off shell photons and gluons and one for the on shell case. For the on shell $Z^{0}$ case, only two form factors survive. The contribution from the third form factor, due to axial current non conservation when quark masses are different from zero, vanishes when the $Z^{0}$ is on shell. We have set to zero the charm mass.

With respect to previous calculations, we take into account the effect of a large left-right stop mixing which produces a large mass splitting and makes the transitions with a gluino helicity flip possible and large when the SUSY 
breaking scale increases.

In the appendix we report the full result for the form factors relevant to the three different decays for the gluino mediated penguins, which represent the dominant contribution when the branching ratios are large.

\section{The results}

In table 11 we report the results for the constrained MSSM for two values of $\tan \beta$ and one chargino masse near the experimental limit, i.e around $90 \mathrm{GeV}$. Squark and gluino masses are set between 200 and $300 \mathrm{GeV}$.

Given the smallness of the mixing angle, the correct result for this case can be obtained already in the mass insertion approximation. For moderate values of $\tan \beta$ the chargino contribution dominates: the gluino contribution becomes comparable only for $\tan \beta=35$. In any case the effect is not visible in the universal SUSY model.

\begin{tabular}{||l|cc|cc||}
\hline & \multicolumn{2}{|c|}{$\tan \beta=2$} & \multicolumn{2}{c||}{$\tan \beta=35$} \\
$B R(t \rightarrow c g)$ & gluino & chargino & gluino & chargino \\
$B R(t \rightarrow c \gamma)$ & $10^{-15}$ & $10^{-11}$ & $10^{-11}$ & $10^{-12}$ \\
$B R\left(t \rightarrow c Z^{0}\right)$ & $10^{-17}$ & $10^{-12}$ & $10^{-13}$ & $10^{-13}$ \\
\hline
\end{tabular}

Table 1: The results of the branching ratios for the MSSM with universal soft breaking.

By abandoning the hypothesis of flavour universality of the soft SUSY breaking terms, one can explore the result for sizeable values of the mixing angles. We have restricted our analysis to the case with a mixing between the second and the third generation only. The most general rotation in this four dimensional space (scharm and stop, both left and right) is parametrized by six angles that we chose as the rotation angles in each of the six possible rotation planes. More explicitely, our rotation matrix $\boldsymbol{R}$ is given by:

$$
\boldsymbol{R}=\boldsymbol{R}^{12} \boldsymbol{R}^{13} \boldsymbol{R}^{14} \boldsymbol{R}^{23} \boldsymbol{R}^{24} \boldsymbol{R}^{34}
$$

where

$$
\boldsymbol{R}^{12}=\left(\begin{array}{cccc}
\cos \theta_{12} & \sin \theta_{12} & 0 & 0 \\
-\sin \theta_{12} & \cos \theta_{12} & 0 & 0 \\
0 & 0 & 1 & 0 \\
0 & 0 & 0 & 1
\end{array}\right)
$$

and similarly for $\boldsymbol{R}^{13}, \boldsymbol{R}^{14}, \boldsymbol{R}^{23}, \boldsymbol{R}^{24}$ and $\boldsymbol{R}^{34}$. We generate uniformly in the six dimensional space of rotation angles a mixing matrix in the basis where the squark mass matrix is diagonal with definite eigenvalues. Our default choice are the values at the lower experimental bounds: 


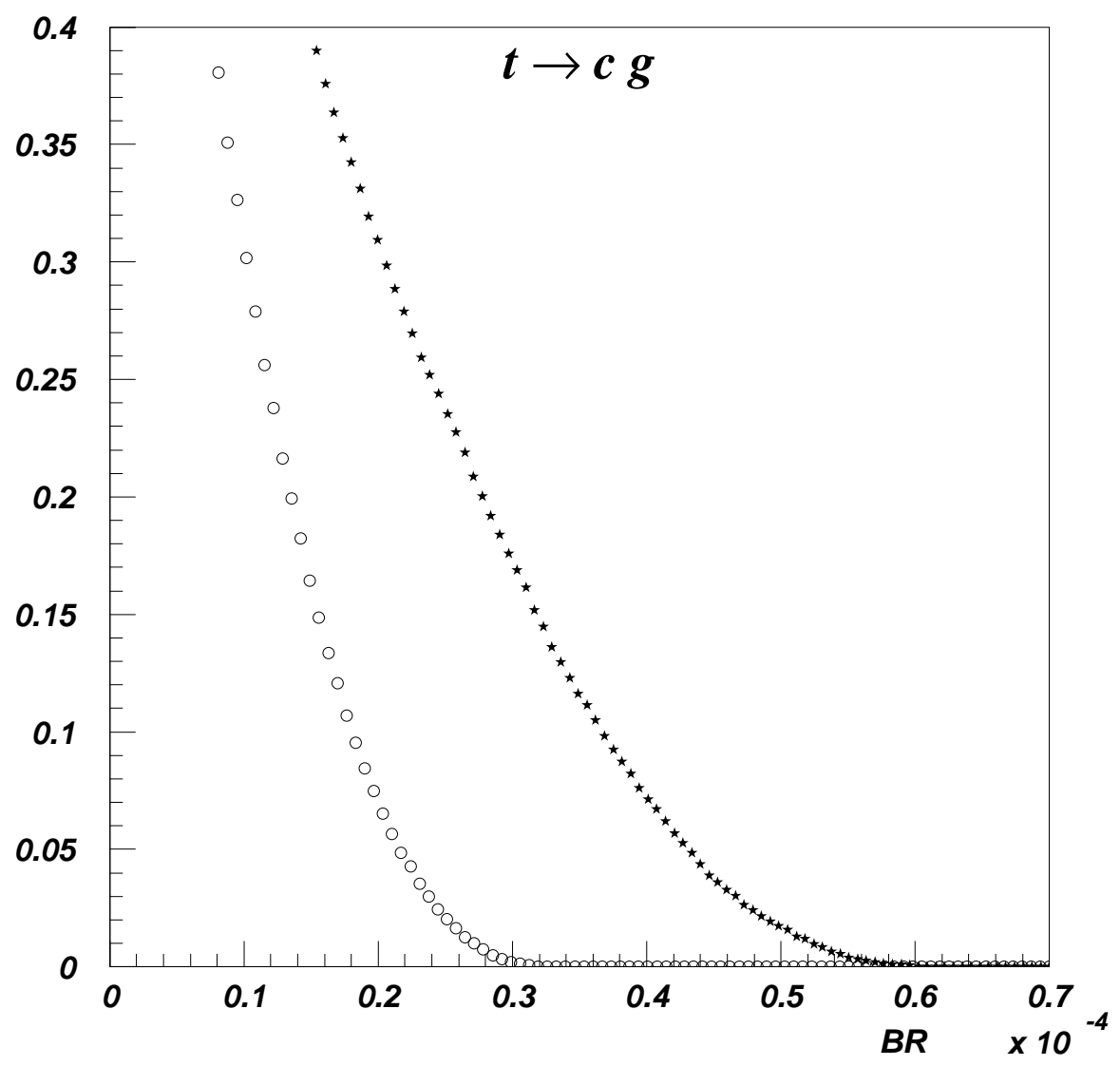

Figure 3: The integral of the events with branching ratio greater than $B R$ generated with random rotation matrix for the $t \rightarrow c g$ decay with gluino penguins for the default choice of squark mass eigenvalues (upper curve) and for a choice with a smaller splitting among the eigenstates (lower curve).

$$
\begin{array}{cl}
m_{\tilde{c}_{1}}=220 \mathrm{GeV}, & m_{\tilde{c}_{2}}=260 \mathrm{GeV}, \\
m_{\tilde{c}_{1}}=90 \mathrm{GeV}, & m_{\tilde{c}_{2}}=180 \mathrm{GeV},
\end{array}
$$

where the labels 1 and 2 denote the proper eigenstates of the mass matrix. The gluino mass has been set equal to $154 \mathrm{GeV}$.

For each choice of the matrix we calculate the branching ratio.

In figure 3 we plot for the gluon decay with intermediate gluino exchange the percentage of the cases where the resulting branching ratio is bigger than a given value. Such a percentage gives an indication about the naturalness of the corresponding values of the branching ratio. The maximum value of the branching ratio is of the order of $5 \cdot 10^{-5}$ and a reasonable percentage of the order of $20 \%$ is obtained for branching ratios larger than 


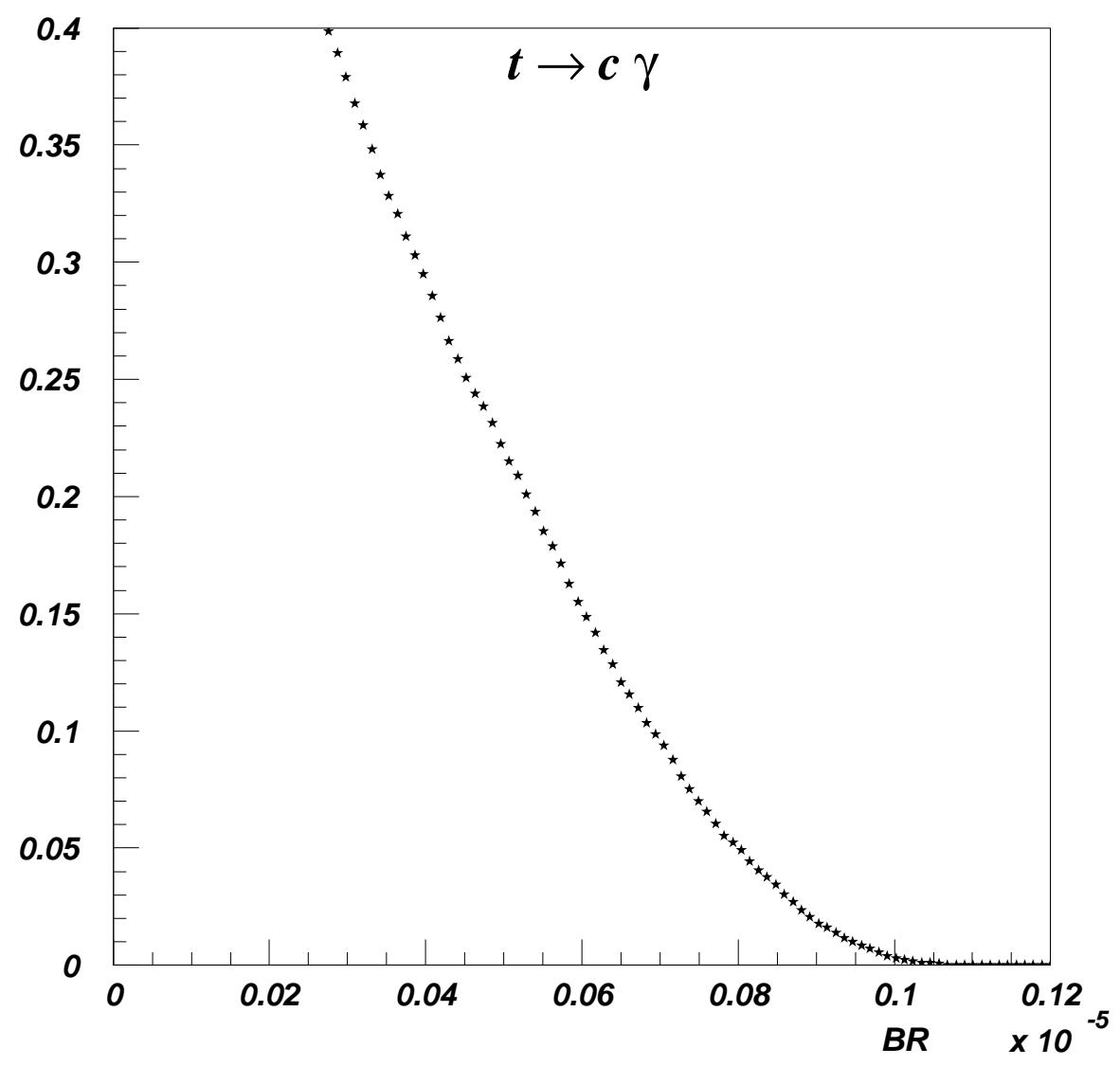

Figure 4: The same as in figure 2 for the $t \rightarrow c \gamma$ decay and the default choice of the mass eigenvalues.

3. $10^{-5}$, i.e. for a detectable signal. On the same figure is given the case where the eigenvalues of stop states have been changed from 90 and 180 to 120 and 160. The curve becomes steeper because of the more severe cancellations due to the GIM mechanism.

Figures 1 and 5 refer to the photon and $Z^{0}$ case, respectively. The values are smaller by a factor ranging from 20 to 50 coming basically from the different colour charge.

In figure 6 we report the variation, for a fixed favourable choice of the mixing angles, of the branching ratio with the values of the squark or of the gluino masses, rescaled by a factor $f$ with respect to their reference values. The dependence upon the gluino mass at fixed default values of the squark masses appears to be more relevant than the reverse case.

For each rotation matrix and choice of the mass eigenvalues we can reconstruct the original non diagonal mass matrix and in particular we can analyse which entries of this matrix should be large to reach the highest values of the branching ratio. This can be seen by plotting, for the events 


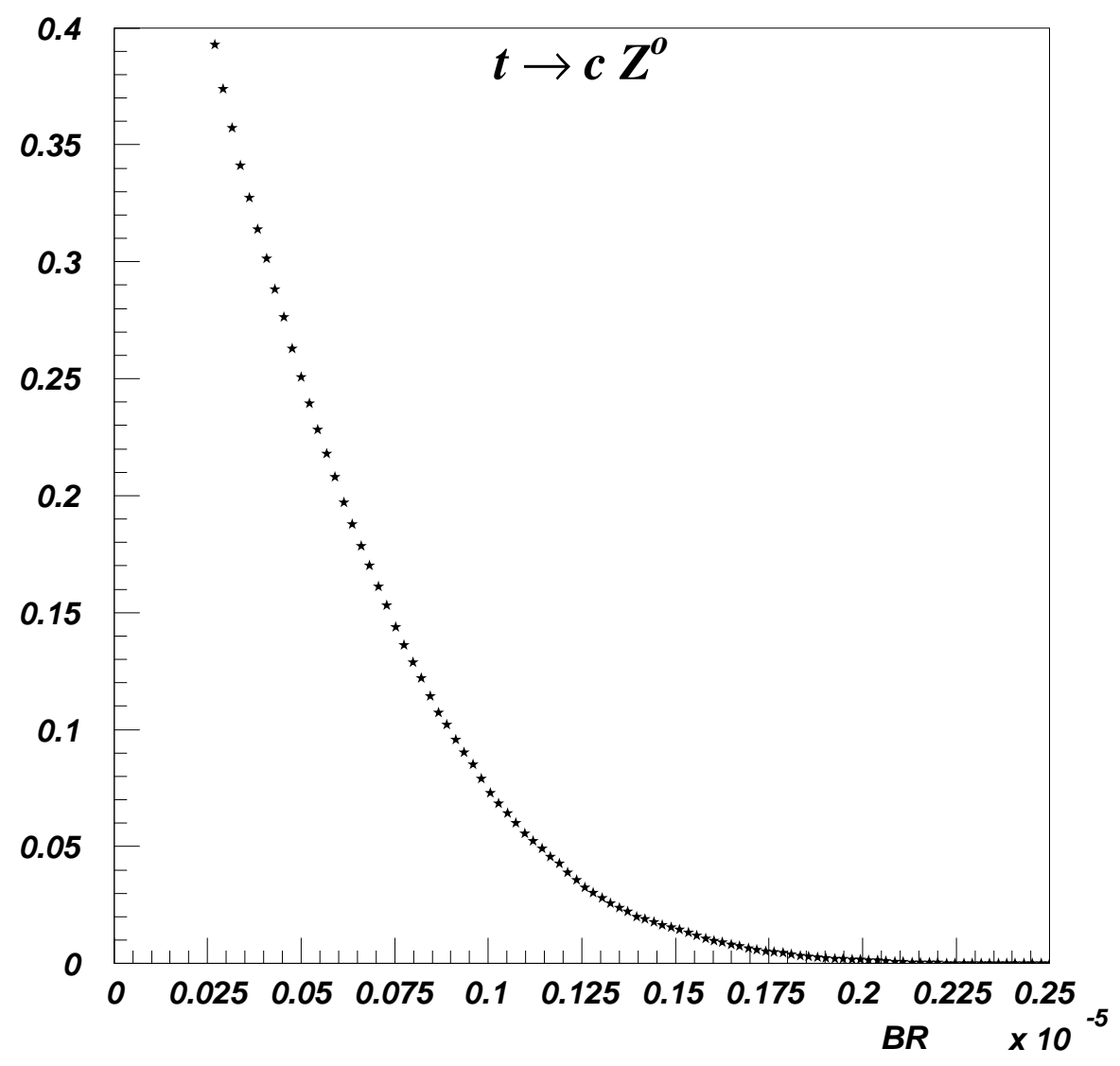

Figure 5: The same as in figure 2 for the $t \rightarrow c Z^{0}$ decay and the default choice of the mass eigenvalues. 


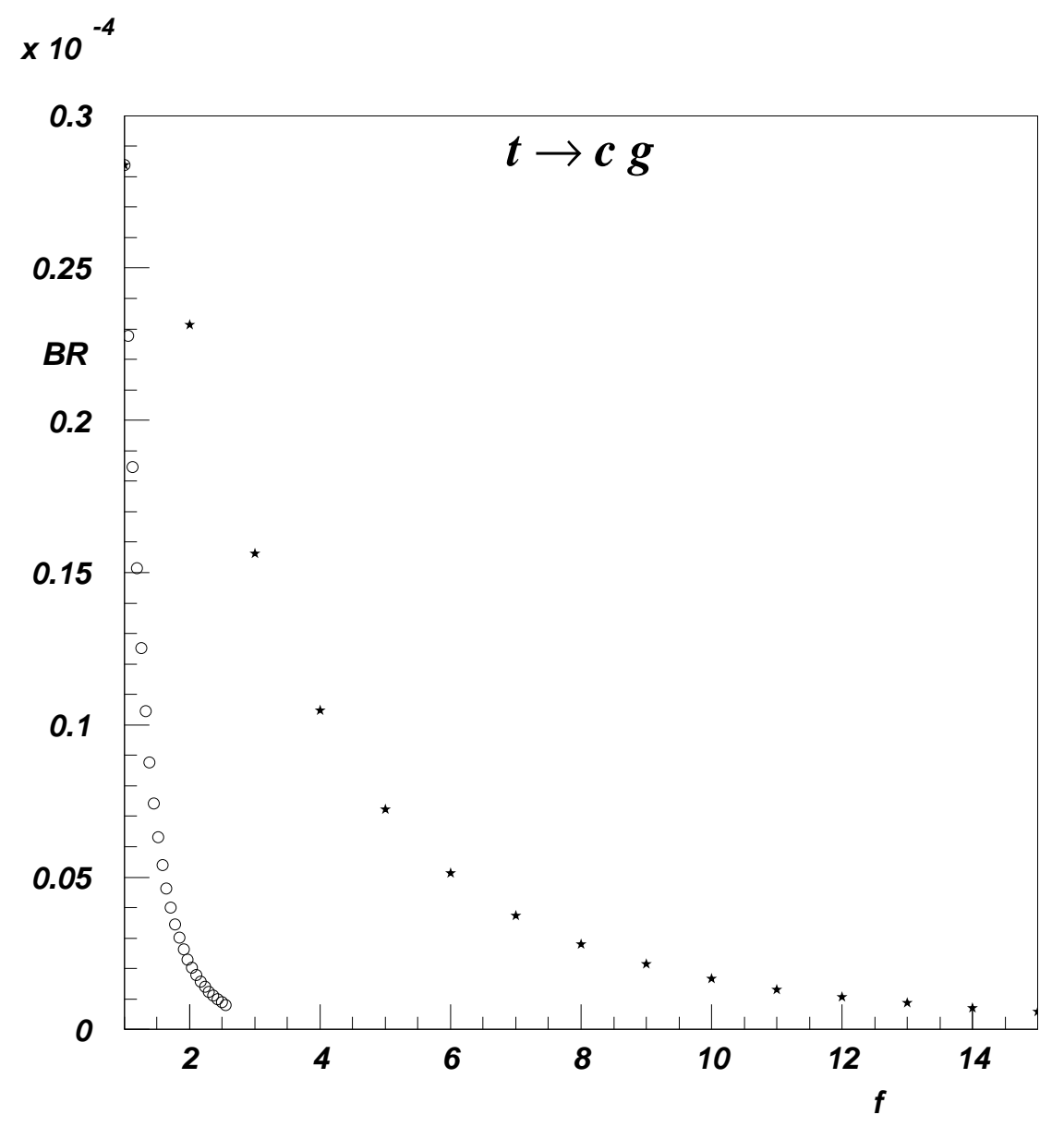

Figure 6: The variation of the branching ratio for a particular and favourable choice of the mixing matrix with the squark (upper curve) or the gluino (lower curve) mass rescaling by a factor $f$. 
generated with a random rotation matrix where the branching ratio is higher than a given threshold value, the correlation of two off diagonal entries of the mass matrix normalized to the average of the diagonal entries.

In figures 0 we plot the correlation of $\tilde{c}_{L}-\tilde{t}_{R}$ versus $\tilde{c}_{R}-\tilde{t}_{L}$ for two different cuts on the branching ratio. Among all possible correlations, only this correlation shows a hole in the center (when both values are close to zero) which increases with the value of the cut. The condition that at least one of the two off diagonal entries $\tilde{c}_{L}-\tilde{t}_{R}$ or $\tilde{c}_{R}-\tilde{t}_{L}$ should be large is a necessary one when a large branching ratio is required. This cannot be realized in the minimal SUSY model with universal couplings.

Flavor changing top decays are expected to be visible only in extensions of the minimal supersymmetric standard model where the soft breaking is flavour dependent and non universal.

NOTE: While completing this work we have seen a paper by Lopez et al., hep-ph 9702350, where the same processes were considered. Their conclusions on the yield of FCNC in SUSY models with universal breaking are different from ours.

\section{Appendix}

We report in this appendix the result for the gluino mediated diagrams.

The effective vertex can be parametrized as follow:

$$
\begin{aligned}
& -i \bar{u}(p)\left\{\quad P_{R}\left(F_{L}^{a} q^{2} \gamma^{\mu}+F_{L}^{b} \not q^{\mu}+G_{L} i \sigma^{\mu \nu} q_{\nu}\right)+\right. \\
& \left.P_{L}\left(F_{R}^{a} q^{2} \gamma^{\mu}+F_{R}^{b} \not q^{\mu}+G_{R} i \sigma^{\mu \nu} q_{\nu}\right)\right\} \epsilon_{\mu} u(p+q)
\end{aligned}
$$

where $P_{L, R}=\frac{\left(1 \mp \gamma_{5}\right)}{2}$ and $\epsilon_{\mu}=\epsilon_{\mu}^{a} \boldsymbol{T}^{a}$ for gluon and $\epsilon_{\mu}=\epsilon_{\mu} \mathbb{I}$ for photon or $Z^{0}$. In the photon and the gluon case gauge invariance implies $F^{a}=-F^{b}$, a relation that we have explicitely checked.

The form factors $F^{a}$ can be written as

$$
\begin{aligned}
& F_{L}^{a}=\frac{g_{s}^{2}}{4 \pi^{2}} \sum_{A, B=1}^{4}\left\{\boldsymbol{R}_{\tilde{c}_{L}, B}^{\dagger} \boldsymbol{R}_{A, \tilde{t}_{L}} F_{1 L}^{a}(A, B)-\boldsymbol{R}_{\tilde{c}_{L}, B}^{\dagger} \boldsymbol{R}_{A, \tilde{t}_{R}} F_{2 L}^{a}(A, B)\right\} \\
& F_{R}^{a}=\frac{g_{s}^{2}}{4 \pi^{2}} \sum_{A, B=1}^{4}\left\{\boldsymbol{R}_{\tilde{c}_{R}, B}^{\dagger} \boldsymbol{R}_{A, \tilde{t}_{R}} F_{1 R}^{a}(A, B)-\boldsymbol{R}_{\tilde{c}_{R}, B}^{\dagger} \boldsymbol{R}_{A, \tilde{t}_{L}} F_{2 R}^{a}(A, B)\right\} .
\end{aligned}
$$

Analogous expressions can be written for $F^{b}$ and $G$. The matrix $\boldsymbol{R}$ diagonalizes the squared squark mass matrix, and the indices $A$ and $B$ identify the eigenvalues and the corresponding eigenvectors:

$$
\left(\begin{array}{c}
\tilde{q}_{1} \\
\tilde{q}_{2} \\
\tilde{q}_{3} \\
\tilde{q}_{4}
\end{array}\right)=\boldsymbol{R}\left(\begin{array}{c}
\tilde{c}_{L} \\
\tilde{c}_{R} \\
\tilde{t}_{L} \\
\tilde{t}_{R}
\end{array}\right) .
$$



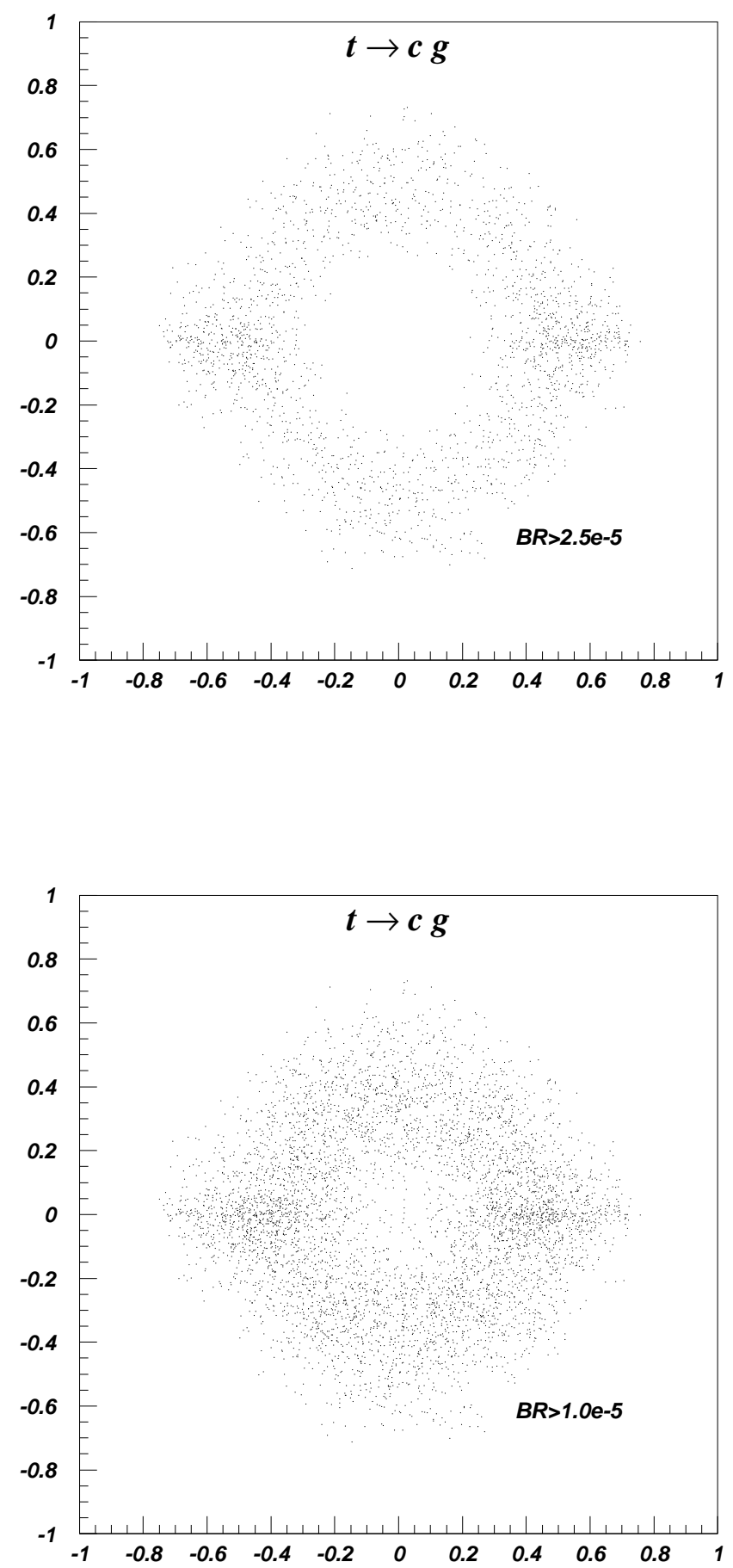

Figure 7: The correlation for the events generated with random rotation matrices of the matrix elements of the original off diagonal squared mass matrix $\tilde{c}_{L}-\tilde{t}_{R}$ and $\tilde{c}_{R}-\tilde{t}_{L}$ for two different cuts of the BR. 
In general $f$ can be split in a piece proportional to $C_{F}=\frac{N^{2}-1}{2 N}=4 / 3$ and one proportional to $C_{G}=N=3$, the latter is present in the gluon case. For the $C_{G}$ terms we report only the expression of the form factor relevant for on shell gluon. For the $C_{F}$ terms we report the result at $q^{2} \neq 0$ which is needed for the $Z^{0}$ case. The form factor $F^{b}$ does not contribute to the branching ratio for on shell vector bosons.

The formulas are parametrized in terms of the following couplings of the gauge boson $V=g, \gamma, Z^{0}$ to the left or right up quarks $a_{L(R)}^{q q V}$ and of the couplings of the gauge boson to the up squarks in the mass eigenstates basis $a_{\tilde{q} \tilde{q} V}(A, B)$ :

$$
\begin{aligned}
\text { for the gluon } & a_{L}^{q q g}=a_{R}^{q q g}=-g_{s}, \\
\text { for the photon } & a_{L}^{q q \gamma}=a_{R}^{q q \gamma}=-g_{e m} \frac{2}{3}, \\
\text { and for } Z^{0} & \\
a_{L}^{q q Z} & =\frac{g_{w}}{2 \cos \theta_{w}}\left(-1+\frac{4}{3} \sin ^{2} \theta_{w}\right) \\
a_{R}^{q q Z} & =\frac{g_{w}}{2 \cos \theta_{w}}\left(\frac{4}{3} \sin ^{2} \theta_{w}\right) .
\end{aligned}
$$

The $Z^{0}$ coupling is different for left and right handed quarks and squarks and therefore is not diagonal in the squark mass eigenstates basis, $a_{\tilde{q} \tilde{q} Z}(A, B)=\left(\boldsymbol{R} \hat{a}_{\tilde{q} \tilde{q} Z} \boldsymbol{R}^{\dagger}\right)_{B A}$ where

$$
\hat{a}_{\tilde{q} \tilde{q} Z}=\operatorname{diag}\left(a_{L}^{q q Z}, a_{R}^{q q Z}, a_{L}^{q q Z}, a_{R}^{q q Z}\right) .
$$

We have used the Feynman rules described in [14. The results have been obtained with algebraic package FORM 15.

For the photon or $Z^{0} F_{. .}^{a}(.,)=.F_{. .}^{a}(., .)^{C_{F}}$ and $G_{. .}(.,)=.G_{. .}(., .)^{C_{F}}$

$$
\begin{aligned}
& F_{1 L(R)}^{a}(A, B)^{C_{F}}= \\
& a_{L(R)}^{q q V} \delta_{A, B} C_{F} \cdot \\
& \frac{1}{2 q^{2}}\left\{B_{x}\left(m_{\text {top }}^{2}, m_{\tilde{g}}^{2}, m_{A}^{2}\right)+B_{0}\left(m_{\text {top }}^{2}, m_{A}^{2}, m_{\tilde{g}}^{2}\right)\right\} \\
& a_{\tilde{q} \tilde{q} V}(A, B) C_{F} \cdot\{ \\
& +\frac{m_{\text {top }}^{2}\left(m_{B}^{2}-m_{\tilde{g}}^{2}\right)}{\left(m_{\text {top }}^{2}-q^{2}\right)^{2} q^{2}}\left\{B_{0}\left(m_{\text {top }}^{2}, m_{\tilde{g}}^{2}, m_{A}^{2}\right)-B_{0}\left(0, m_{\tilde{g}}^{2}, m_{B}^{2}\right)-\left(m_{A}^{2}-m_{B}^{2}\right) C_{0}\left(m_{A}^{2}, m_{\tilde{g}}^{2}, m_{B}^{2}\right)\right\} \\
& +\frac{3}{2} \frac{m_{\text {top }}^{2}\left(m_{B}^{2}-m_{\tilde{g}}^{2}\right)}{\left(m_{\text {top }}^{2}-q^{2}\right)^{3}}\left\{B_{0}\left(m_{\text {top }}^{2}, m_{\tilde{g}}^{2}, m_{A}^{2}\right)-B_{0}\left(q^{2}, m_{B}^{2}, m_{A}^{2}\right)+\left(m_{B}^{2}-m_{\tilde{g}}^{2}\right) C_{0}\left(m_{A}^{2}, m_{\tilde{g}}^{2}, m_{B}^{2}\right)\right\} \\
& -\frac{1}{2} \frac{m_{t o p}^{2}}{\left(m_{\text {top }}^{2}-q^{2}\right) q^{2}}\left\{B_{0}\left(m_{\text {top }}^{2}, m_{\tilde{g}}^{2}, m_{A}^{2}\right)+B_{x}\left(m_{\text {top }}^{2}, m_{A}^{2}, m_{\tilde{g}}^{2}\right)\right\} \\
& -\frac{1}{2} \frac{\left(m_{B}^{2}-m_{\tilde{g}}^{2}\right)}{\left(m_{t o p}^{2}-q^{2}\right)^{2}}\left\{B_{0}\left(m_{\text {top }}^{2}, m_{\tilde{g}}^{2}, m_{A}^{2}\right)-B_{0}\left(q^{2}, m_{A}^{2}, m_{B}^{2}\right)+\left(m_{B}^{2}-m_{\tilde{g}}^{2}\right) C_{0}\left(m_{A}^{2}, m_{\tilde{g}}^{2}, m_{B}^{2}\right)\right\} \\
& -\frac{1}{2} \frac{m_{\text {top }}^{2}}{\left(m_{\text {top }}^{2}-q^{2}\right)^{2}}\left\{B_{x}\left(m_{\text {top }}^{2}, m_{A}^{2}, m_{\tilde{g}}^{2}\right)-B_{x}\left(q^{2}, m_{A}^{2}, m_{B}^{2}\right)-2\left(m_{B}^{2}-m_{\tilde{g}}^{2}\right) C_{0}\left(m_{A}^{2}, m_{\tilde{g}}^{2}, m_{B}^{2}\right)\right\} \\
& -\frac{1}{2} \frac{1}{\left(m_{\text {top }}^{2}-q^{2}\right)}\left\{B_{x}\left(q^{2}, m_{A}^{2}, m_{B}^{2}\right)-m_{\tilde{g}}^{2} C_{0}\left(m_{A}^{2}, m_{\tilde{g}}^{2}, m_{B}^{2}\right)-\frac{1}{2}\right\}
\end{aligned}
$$




$$
\begin{aligned}
& \left.-\frac{1}{2} \frac{\left(m_{B}^{2}-m_{\tilde{g}}^{2}\right)}{\left(m_{\text {top }}^{2}-q^{2}\right) q^{2}}\left\{B_{0}\left(m_{\text {top }}^{2}, m_{\tilde{g}}^{2}, m_{A}^{2}\right)-B_{0}\left(0, m_{\tilde{g}}^{2}, m_{B}^{2}\right)-\left(m_{A}^{2}-m_{B}^{2}\right) C_{0}\left(m_{A}^{2}, m_{\tilde{g}}^{2}, m_{B}^{2}\right)\right\}\right\} \\
& F_{2 L(R)}^{a}(A, B)^{C_{F}}= \\
& a_{L(R)}^{q q V} \delta_{A, B} C_{F} \cdot \\
& \left.\frac{1}{2} \frac{m_{\text {top }} m_{\tilde{g}}}{m_{\text {top }}^{2} q^{2}}\left\{B_{0}\left(m_{\text {top }}^{2}, m_{\tilde{g}}^{2}, m_{A}^{2}\right)-B_{0}\left(0, m_{A}^{2}, m_{\tilde{g}}^{2}\right)\right\}\right\} \\
& a_{\tilde{q} \tilde{q} V}(A, B) C_{F} \cdot\{ \\
& -\frac{1}{2} \frac{m_{\text {top }} m_{\tilde{g}}}{\left(m_{\text {top }}^{2}-q^{2}\right) q^{2}}\left\{B_{0}\left(m_{\text {top }}^{2}, m_{\tilde{g}}^{2}, m_{A}^{2}\right)-B_{0}\left(0, m_{\tilde{g}}^{2}, m_{B}^{2}\right)-\left(m_{A}^{2}-m_{B}^{2}\right) C_{0}\left(m_{A}^{2}, m_{\tilde{g}}^{2}, m_{B}^{2}\right)\right\} \\
& -\frac{m_{\text {top }} m_{\tilde{g}}}{\left(m_{\text {top }}^{2}-q^{2}\right)^{2}}\left\{B_{0}\left(m_{\text {top }}^{2}, m_{\tilde{g}}^{2}, m_{A}^{2}\right)-B_{0}\left(q^{2}, m_{B}^{2}, m_{A}^{2}\right)+\left(m_{B}^{2}-m_{\tilde{g}}^{2}\right) C_{0}\left(m_{A}^{2}, m_{\tilde{g}}^{2}, m_{B}^{2}\right)\right\} \\
& \left.-\frac{1}{2} \frac{m_{t o p} m_{\tilde{g}}}{\left(m_{\text {top }}^{2}-q^{2}\right)}\left\{C_{0}\left(m_{A}^{2}, m_{\tilde{g}}^{2}, m_{B}^{2}\right)\right\}\right\}
\end{aligned}
$$

It can be verified that the above expression has a smooth limit when $q^{2} \rightarrow 0$.

$$
\begin{aligned}
& G_{1 L}(A, B)^{C_{F}}=G_{1 R}(A, B)^{C_{F}}= \\
& a_{\tilde{q} \tilde{q} V}(A, B) C_{F} \cdot\{ \\
& +m_{\text {top }} \frac{\left(m_{B}^{2}-m_{\tilde{g}}^{2}\right)}{\left(m_{\text {top }}^{2}-q^{2}\right)^{2}}\left\{B_{0}\left(m_{\text {top }}^{2}, m_{\tilde{g}}^{2}, m_{A}^{2}\right)-B_{0}\left(0, m_{\tilde{g}}^{2}, m_{B}^{2}\right)-\left(m_{A}^{2}-m_{B}^{2}\right) C_{0}\left(m_{A}^{2}, m_{\tilde{g}}^{2}, m_{B}^{2}\right)\right\} \\
& +\frac{3}{2} \frac{q^{2} m_{\text {top }}\left(m_{B}^{2}-m_{\tilde{g}}^{2}\right)}{\left(m_{\text {top }}^{2}-q^{2}\right)^{3}}\left\{B_{0}\left(m_{\text {top }}^{2}, m_{\tilde{g}}^{2}, m_{A}^{2}\right)-B_{0}\left(q^{2}, m_{B}^{2}, m_{A}^{2}\right)+\left(m_{B}^{2}-m_{\tilde{g}}^{2}\right) C_{0}\left(m_{A}^{2}, m_{\tilde{g}}^{2}, m_{B}^{2}\right)\right\} \\
& -\frac{1}{2} \frac{m_{\text {top }} q^{2}}{\left(m_{\text {top }}^{2}-q^{2}\right)^{2}}\left\{B_{x}\left(m_{\text {top }}^{2}, m_{A}^{2}, m_{\tilde{g}}^{2}\right)-B_{x}\left(q^{2}, m_{A}^{2}, m_{B}^{2}\right)-2\left(m_{B}^{2}-m_{\tilde{g}}^{2}\right) C_{0}\left(m_{A}^{2}, m_{\tilde{g}}^{2}, m_{B}^{2}\right)\right\} \\
& \left.-\frac{1}{2} \frac{m_{\text {top }}}{\left(m_{\text {top }}^{2}-q^{2}\right)}\left\{B_{0}\left(m_{t o p}^{2}, m_{A}^{2}, m_{\tilde{g}}^{2}\right)+2 B_{x}\left(m_{\text {top }}^{2}, m_{A}^{2}, m_{\tilde{g}}^{2}\right)-m_{B}^{2} C_{0}\left(m_{A}^{2}, m_{\tilde{g}}^{2}, m_{B}^{2}\right)-\frac{1}{2}\right\}\right\} \\
& G_{2 L}(A, B)^{C_{F}}=G_{2 R}(A, B)^{C_{F}}= \\
& a_{\tilde{q} \tilde{q} V}(A, B) C_{F} \cdot\{ \\
& -\frac{1}{2} \frac{m_{\tilde{g}}}{\left(m_{t o p}^{2}-q^{2}\right)}\left\{B_{0}\left(m_{t o p}^{2}, m_{\tilde{g}}^{2}, m_{A}^{2}\right)-B_{0}\left(0, m_{\tilde{g}}^{2}, m_{B}^{2}\right)-\left(m_{A}^{2}-m_{B}^{2}\right) C_{0}\left(m_{A}^{2}, m_{\tilde{g}}^{2}, m_{B}^{2}\right)\right\} \\
& -\frac{m_{\tilde{g}} q^{2}}{\left(m_{\text {top }}^{2}-q^{2}\right)^{2}}\left\{B_{0}\left(m_{t o p}^{2}, m_{\tilde{g}}^{2}, m_{A}^{2}\right)-B_{0}\left(q^{2}, m_{B}^{2}, m_{A}^{2}\right)+\left(m_{B}^{2}-m_{\tilde{g}}^{2}\right) C_{0}\left(m_{A}^{2}, m_{\tilde{g}}^{2}, m_{B}^{2}\right)\right\} \\
& \left.-\frac{1}{2} \frac{q^{2} m_{\tilde{g}}}{\left(m_{t o p}^{2}-q^{2}\right)}\left\{C_{0}\left(m_{A}^{2}, m_{\tilde{g}}^{2}, m_{B}^{2}\right)\right\}\right\}
\end{aligned}
$$

For the gluon $G_{. .}(.,)=.G_{. .}(., .)^{C_{F}}+G_{. .}(., .)^{C_{G}}$

$$
\begin{aligned}
& G_{1 L}(A, B)^{C_{G}}=G_{1 R}(A, B)^{C_{G}}= \\
& -g_{s} \delta_{A, B} \frac{1}{2} C_{G} \cdot\{ \\
& -m_{\text {top }} \frac{\left(m_{B}^{2}-m_{\tilde{g}}^{2}\right)}{\left(m_{\text {top }}^{2}\right)^{2}}\left\{B_{0}\left(m_{\text {top }}^{2}, m_{\tilde{g}}^{2}, m_{A}^{2}\right)-B_{0}\left(0, m_{\tilde{g}}^{2}, m_{B}^{2}\right)-\left(m_{A}^{2}-m_{B}^{2}\right) C_{0}\left(m_{A}^{2}, m_{\tilde{g}}^{2}, m_{B}^{2}\right)\right\} \\
& +\frac{1}{2} \frac{m_{\text {top }}}{m_{\text {top }}^{2}}\left\{B_{0}\left(m_{\text {top }}^{2}, m_{\tilde{g}}^{2}, m_{A}^{2}\right)+2 B_{x}\left(m_{\text {top }}^{2}, m_{A}^{2}, m_{\tilde{g}}^{2}\right)-m_{B}^{2} C_{0}\left(m_{A}^{2}, m_{\tilde{g}}^{2}, m_{B}^{2}\right)-\frac{1}{2}\right\} \\
& +m_{\text {top }} \frac{\left(m_{A}^{2}-m_{\tilde{g}}^{2}\right)}{\left(m_{\text {top }}^{2}\right)^{2}}\left\{B_{0}\left(m_{\text {top }}^{2}, m_{\tilde{g}}^{2}, m_{A}^{2}\right)-B_{0}\left(0, m_{\tilde{g}}^{2}, m_{A}^{2}\right)\right\} \\
& \left.+\frac{1}{2} \frac{m_{\text {top }}}{m_{\text {top }}^{2}}\left\{B_{0}\left(m_{\text {top }}^{2}, m_{\tilde{g}}^{2}, m_{A}^{2}\right)+2 B_{x}\left(m_{\text {top }}^{2}, m_{\tilde{g}}^{2}, m_{A}^{2}\right)-m_{\tilde{g}}^{2} C_{0}\left(m_{\tilde{g}}^{2}, m_{A}^{2}, m_{\tilde{g}}^{2}\right)-\frac{1}{2}\right\}\right\}
\end{aligned}
$$




$$
\begin{aligned}
& G_{2 L}(A, B)^{C_{G}}=G_{2 R}(A, B)^{C_{G}}= \\
& -g_{s} \delta_{A, B} \frac{1}{2} C_{G} \cdot\{ \\
& +\frac{1}{2} \frac{m_{\tilde{g}}}{m_{\text {top }}^{2}}\left\{B_{0}\left(m_{\text {top }}^{2}, m_{\tilde{g}}^{2}, m_{A}^{2}\right)-B_{0}\left(0, m_{\tilde{g}}^{2}, m_{B}^{2}\right)-\left(m_{A}^{2}-m_{B}^{2}\right) C_{0}\left(m_{A}^{2}, m_{\tilde{g}}^{2}, m_{B}^{2}\right)\right\} \\
& \left.-\frac{1}{2} \frac{m_{\tilde{g}}}{m_{\text {top }}^{2}}\left\{B_{0}\left(m_{\text {top }}^{2}, m_{\tilde{g}}^{2}, m_{A}^{2}\right)-B_{0}\left(0, m_{\tilde{g}}^{2}, m_{A}^{2}\right)+m_{\text {top }}^{2} C_{0}\left(m_{\tilde{g}}^{2}, m_{A}^{2}, m_{\tilde{g}}^{2}\right)\right\}\right\}
\end{aligned}
$$

Where:

$$
\begin{aligned}
& B_{0}\left(p^{2}, m_{1}^{2}, m_{2}^{2}\right)=\text { pole terms }+ \\
& \quad-\int_{0}^{1} d \alpha \log \left(p^{2} \alpha^{2}+\left(m_{1}^{2}-m_{2}^{2}-p^{2}\right) \alpha+m_{2}^{2}\right) \\
& B_{x}\left(p^{2}, m_{1}^{2}, m_{2}^{2}\right)=\text { pole terms }+ \\
& \quad+\int_{0}^{1} d \alpha \alpha \log \left(p^{2} \alpha^{2}+\left(m_{1}^{2}-m_{2}^{2}-p^{2}\right) \alpha+m_{2}^{2}\right) \\
& C_{0}\left(m_{1}^{2}, m_{2}^{2}, m_{3}^{2}\right)= \\
& \quad \int_{0}^{1} d \alpha \frac{1}{\left(m_{\text {top }}^{2}-q^{2}\right) \alpha+\left(m_{3}^{2}-m_{2}^{2}\right)} \log \frac{\left(m_{\text {top }}^{2} \alpha^{2}+\left(m_{1}^{2}-m_{2}^{2}-m_{\text {top }}^{2}\right) \alpha+m_{2}^{2}\right)}{\left(q^{2} \alpha^{2}+\left(m_{1}^{2}-m_{3}^{2}-q^{2}\right) \alpha+m_{3}^{2}\right)}
\end{aligned}
$$

The above integrals contain divergent parts (pole terms) which cancel in the finite result because the matrix $\boldsymbol{R}$ is unitary.

The integrals are performed in the dimensional regularization scheme: in particular the reduction of tensor integrals to scalar integrals is performed in $4-\epsilon$ dimensions, while the gamma matrices are kept in 4 dimension. The final result being finite is indipendent upon the particular regularization that we have chosen.

In terms of the above form factors the expression for the branching ratios reads:

$$
\begin{aligned}
& B R=\frac{\Gamma(t \rightarrow c+V)}{\Gamma(t \rightarrow b+W)} \quad \text { with } \Gamma(t \rightarrow b+W) \sim 1.52 G e V \text { and } \\
& \Gamma(t \rightarrow c+\gamma)=\left.\frac{1}{16 \pi} m_{\text {top }}^{3}\left(G_{L}^{2}+G_{R}^{2}\right)\right|_{\left(q^{2}=0\right)} \\
& \Gamma(t \rightarrow c+g)=\left.\frac{1}{16 \pi} C_{F} m_{\text {top }}^{3}\left(G_{L}^{2}+G_{R}^{2}\right)\right|_{\left(q^{2}=0\right)} \\
& \Gamma\left(t \rightarrow c+Z^{0}\right)= \frac{1}{32 \pi} \frac{m_{\text {top }}^{2}-m_{Z}^{2}}{2 m_{\text {top }}^{3}} . \\
&\left\{\left.\left(F_{L}^{a 2}+F_{R}^{a 2}\right)\right|_{\left(q^{2}=m_{Z}^{2}\right)}\left(2 m_{\text {top }}^{4} m_{Z}^{2}+2 m_{\text {top }}^{2} m_{Z}^{4}-4 m_{Z}^{6}\right)\right. \\
&+\left.\left(F_{L}^{a} G_{L}+F_{R}^{a} G_{R}\right)\right|_{\left(q^{2}=m_{Z}^{2}\right)}\left(-12 m_{\text {top }}^{3} m_{Z}^{2}+12 m_{\text {top }} m_{Z}^{4}\right) \\
&\left.+\left.\left(G_{L}^{2}+G_{R}^{2}\right)\right|_{\left(q^{2}=m_{Z}^{2}\right)}\left(4 m_{\text {top }}^{4}-2 m_{\text {top }}^{2} m_{Z}^{2}-2 m_{Z}^{4}\right)\right\}
\end{aligned}
$$




\section{References}

[1] H. Fritzsch, Phys. Lett. B224 (1989) 423;

G. Eilam, J. L. Hewett and A. Soni, Phys. Rev. D44 (1991) 1473;

B. Grzadkowski, J. F. Gunion and P. Krawczyk, Phys. Lett. B268 (1991) 106;

N. G. Deshpande, B. Margolis, and H. Trottier, Phys. Rev. D45 (1992) 178 ;

M. Luke and M. Savage, Phys. Lett. B307 (1993) 387;

D. Atwood, L. Reina and A. Soni, hep-ph/9609279.

[2] C. S. Li, R. J. Oakes and J. M. Yang, Phys. Rev. D49 (1994) 293.

[3] G. Couture, C. Hamzaoui, and H. Konig, Phys. Rev. D52 (1995) 1713.

[4] J.-P. Derendinger and C.A. Savoy, Nucl. Phys. 237 (1984) 307.

[5] A. Bouquet, J. Kaplan and C.A. Savoy, Phys. Lett. 148 (1984) 69.

[6] S. Bertolini, F. Borzumati, A. Masiero and G. Ridolfi, Nucl. Phys. B353 (1991) 591.

[7] L.J. Hall, V.A. Kostelecki and S. Raby, Nucl. Phys. B267 (1986) 415.

[8] F. Gabbiani and A. Masiero, Nucl. Phys. B322 (1989) 235.

[9] J. Hagelin, S. Kelley, and T. Tanaka, Nucl. Phys. B415 (1994) 293.

[10] E. Gabrielli, A. Masiero, and L. Silvestrini, Phys. Lett. B374 (1996) 80.

[11] F. Gabbiani, E. Gabrielli, A. Masiero and L. Silvestrini, Nucl. Phys. B477 (1996) 321.

[12] E. Gabrielli, A. Masiero, M. Misiak, S. Pokorski, J. Rosiek and L. Silvestrini, in preparation.

[13] M. J. Duncan, Nucl. Phys. B221 (1983) 221;

M. J. Duncan, Phys. Rev. D31 (1985) 1139.

[14] H. E. Haber and G. L. Kane, Phys. Rep. 117 (1985) 75;

J. F. Gunion and H. E. Haber, Nucl. Phys. 272 (1986) 1.

[15] FORM version 1.0, a symbolic manipulation program by J. Vermaseren; http://www.nikhef.nl/pub/theory. 